\title{
Addressing Menstruation in the Workplace: The Menstrual Leave Debate
}

\author{
Rachel B. Levitt and Jessica L. Barnack-Taplaris
}

\begin{abstract}
INTRODUCTION
Menstrual stigma socially conditions menstruators ${ }^{1}$ to conceal menstruation and avoid discussion about it (Johnston-Robledo and Chrisler 2013, 11). Both concealment and secrecy can have detrimental consequences for a menstruator's psychological well-being, such as holding negative attitudes toward menstruation and partaking in self-objectification (Johnston-Robledo and Chrisler 2013; Roberts, Calogero, and Gervais 2018). The oppressive beliefs about and attitudes toward menstruation that permeate heteropatriarchal culture can extend beyond menstruators' psychological health; they may negatively affect their overall well-being including their personal and professional achievements and success, physical health, and the right to feel empowered and experience equality.

Menstrual leave, which allows a menstruator to take time off if they are unable to attend work due to menstruation, is a policy that could affect menstruators in many ways, including their status in the workplace. The question is whether such a policy would benefit or disadvantage a menstruator's well-being. In spite of progressive aims, could menstrual leave policies actually increase discrimination and negative attitudes toward menstruators?

Proponents of menstrual leave argue that such policies have the potential to de-stigmatize discussion of menstruation in the workplace (CBC Radio 2017), and may be helpful to those who experience menstrual cycle-related illnesses such as endometriosis and dysmenorrhea. However, there is a paucity of research on the effects of these policies on menstruators and menstrual stigma, as well as on the places of employment that are offering them. Furthermore, there is no research to date on the ways in which menstrual leave may be counterproductive to the overall welfare of menstruators through
\end{abstract}


unintended consequences, such as discrimination in hiring practices, salaries or wages, and promotion. Thus, the purpose of this chapter is to (1) present the controversy surrounding menstrual leave through discussion of the potential implications of such policies, (2) argue that without thoughtful implementation, menstrual leave could have negative effects on menstruators, and (3) highlight the gaps and call for more research in this area. Throughout the chapter, we integrate findings from a study we conducted with a national U.S. sample, which examined attitudes toward and perceptions of menstrual leave (Barnack-Tavlaris et al. 2019).

\section{Existing Menstrual Leave Policies}

To date, menstrual leave policies exist across the globe in places such as Japan, Taiwan, China, South Korea, Indonesia, Zambia, and Mexico (Chang et al. 2011; Dan 1986; Forster 2016; Matchar 2014; Worley 2017). Additionally, some professional organizations/companies have chosen to implement menstrual leave, including Coexist (U.K.), Culture Machine (India), Gozoop (India), and the Victorian Women's Trust (Australia). It is difficult to ascertain how these policies are implemented and the extent to which women use the leave because there is often little public data and limited access to human resources policies and procedures which companies typically do not make publically available.

Japan's national policy was introduced in 1947 after American Occupation Forces advised the country to provide women with days off during menstruation (Dan 1986, 8). Japan's menstrual leave policy allows any female worker to use leave if she experiences physical distress from menstruation so severe it makes it difficult to attend work (Dan 1986, 1). The policy does not specify the number of days or whether the leave is paid or unpaid (Dan 1986, 1; Japanese Labor Standards Act 1947). Dan found that women's divisions of labor unions pushed for paid menstrual leave because "they argued that menstruation is a 'barometer' for reproductive ability, and that even women without symptoms ought to take leave to protect their future motherhood" (Dan 1986, 8). However, women who used leave for this purpose were faced with negative consequences such as discrimination and harassment by employers. This resulted in the government proposing to remove menstrual leave from the law to safeguard female workers from discrimination, and led to tension between women's labor unions and the government (Dan 1986, 9-11). While many working women supported the policy in principle, use of the policy varied greatly. Many women rarely used leave due to a variety of factors, including a lack of need, improvements in working conditions (that is, less physical labor demands and more paid holidays), the fact that menstrual leave was unpaid for non-union workers, and making the choice between creating a work life and potential motherhood (Dan 1986, 9). Due to a lack of publicly available data, it is unclear to which extent menstrual leave is presently used among Japan's menstruating population. 
Publicly available information about menstrual leave policies in other countries (mostly in Asia) is limited. Three provinces in China (Hubei, Shanxi, and Ningxia) currently offer menstrual leave (Worley 2017), and in Indonesia, women are allotted two days per month of menstrual leave (Matchar 2014). In 2001, South Korean female workers were granted one day of menstrual leave per month (Matchar 2014). Additionally, a menstrual leave amendment was added to Taiwan's Act of Gender Equality in Employment in 2002, which allotted female employees the right to apply for menstrual leave (Chang et al. 2011).

In Zambia, women are given one menstrual leave day per month. The policy is referred to as a "Mother's Day," which stresses women's potential of becoming mothers (Worley 2017). In 2017, Italy proposed a bill for a menstrual leave policy to parliament which would ensure that companies grant three paid days per month to female employees who experience painful periods, as long as they provide a medical certificate from a doctor (Momigliano 2017). In Mexico, women working in the federal court are eligible for one day of menstrual leave if they experience physical complications, however, the

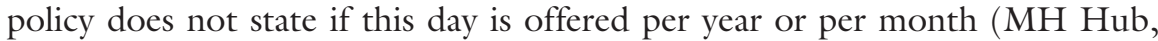
n.d.a). In Chile, a bill is being proposed to allot women paid leave if they have been diagnosed with endometriosis and/or dysmenorrhea (MH Hub, n.d.b).

At the level of companies and organizations, Coexist, a social enterprise organization in the U.K., with apparent progressive values, offers their female workers the option of one paid day of menstrual leave per month (Quarshie 2017). Bex Baxter, Coexist's former People Development Manager, dealt with dysmenorrhea for years before developing a flexible menstrual leave policy that would support Coexist's female staff (Quarshie 2017). The policy was developed together with Lara Owen, who explains that "Bex and her Board felt it was important to formalise the policy to legitimise and think through women's needs at work, and perhaps also to act as a model and inspiration for other organisations to do the same" (Owen 2018, 26). The following statement appears in an online FAQ about Coexist's policy

Menstruating staff who opt into the policy are entrusted to respect their cycle and take responsibility for their own well-being. . . they need to check in with their line manager regarding their individual well-being requirements, and in any instance, any time off or alteration to their working hours must be communicated and signed off with their manager. Some roles allow menstruating staff the option to work from home, or alternatively to use a quiet space away from the main office. . Coexist recognises the importance of difference and debate, therefore should an employee not wish to take part in the policy, they can request to opt out with no judgement or discussion. (Quarshie 2017)

Coexist also offers all of their employees "well-being rooms" where menstruators and non-menstruators alike can take a moment to focus on their health and wellness during work hours (Quarshie 2017). As of 2017, 7 out 
of 13 menstruating employees have used the menstrual leave policy (Quarshie 2017). Additionally, the company asserts that there was no backlash from men. They report that "men expressed no resentment, and instead said they liked the fact that menstruation was addressed openly, and felt it gave them permission to also adjust their working day to their bodies when needed" (Owen 2018, 28).

In India, two companies offer their employees menstrual leave. Gozoop, a digital communications agency, first introduced their policy in 2017. This policy states that women may work from home one day per month during menstruation.

The thought behind this policy is that a woman needs to rest her body while on her period, so she has the facility to work from her comfort zone. We take away the stress of travel, crowded environments, uncomfortable chairs, fear of stains, etc. which will enable women to complete work in an efficient and effective manner from home. While drafting this policy we particularly ensured for it to be a work from home so that this does not make the gender less hire-able. . . About $76 \%$ of the (female) workforce have used their menstrual leave since implemented in March 2017. (Bansi Raja, Email to author, June 7, 2018)

This policy differs from other menstrual leave policies in that it offers flexibility in the workplace instead of actual time off. For example, employees are able to work from home instead of in the office. Another company, Culture Machine, a digital media firm, offers their female employees one paid day off per month, referred to as 'First Day of Period Leave' (Blush Originals 2017). The company created a YouTube video that showed female employees finding out about their menstrual leave policy for the first time. Women in the video were excited about the policy and felt that it would contribute positively to female employees' well-being (Blush Originals 2017).

In Australia, the Victorian Women's Trust (VWT), a women's advocacy agency with 15 employees, offers their staff a menstrual policy that extends to employees in menopause (the first of its kind); furthermore, they have created a policy template to encourage other companies to offer menstrual leave (Melican and Mountford 2017). Their policy began with the Waratah Project, which explored issues surrounding menstruation and menopause in order to end stigma (Melican and Mountford 2017). In an online survey, they found that $58 \%$ of respondents across Australia and globally supported the idea of menstrual leave, believing that it would contribute to a better menstrual experience (Melican and Mountford 2017, 1). VWT's menstrual leave policy provides employees with multiple options, including working from home, working in the office in a more quiet and comfortable area, or using one paid menstrual leave day per month (Melican and Mountford 2017). The VWT hopes that their policy will send the message that menstruation and menopause are natural and normal biological processes (Melican and Mountford 2017). 
Given the existence of diverse menstrual leave policies across the globe and the current discussion of whether or not to extend such policies, it is important to think critically about both the potential benefits and drawbacks that could result from implementing menstrual leave. In these next sections, we will explore this tension, incorporate some findings from our qualitative study that speak to these potential outcomes, and highlight that more research is needed in order to determine the full extent of the implications of menstrual leave on menstruators' overall well-being and status in the workplace. Further, we argue that the potential impact of these policies will be determined by how and where they are implemented.

\section{Theorizing Menstrual Leave: Using the Framework of Objectification and Ambivalent Sexism}

The discussion on menstrual leave must be understood in the context of societies in which menstruation has been used to marginalize and oppress women and other menstruators through the process of objectification and the ideology of sexism. Objectification dehumanizes women by reducing their worth to the surface of their bodies (Bordo 1993, 309; Fredrickson and Roberts 1997). Objectification is achieved under the male gaze, an invisible yet unavoidable presence that encompasses heteropatriarchal, and often Eurocentric, views of how women are expected to behave and appear that disassociates women from their own well-being, success, needs, and desires (Bartky 1988; Roberts, Calogero, and Gervais 2018; Roberts et al. 2002). In order to preserve status in a heteropatriarchal society, women must internalize the male gaze and practice self-monitoring body disciplines such as dieting, makeup, the use of menstrual care products (often advertised as "feminine hygiene products"), and by concealing all matters of menstruation (Bartky 1988; Johnston-Robledo and Chrisler 2013; Roberts et al. 2002). Researchers have found that in order for women to meet the unrealistic standards of femininity, they must choose to "adopt a menstrual etiquette that reinforces fear about the female body and its functions" (Grose and Grabe 2014, 679). As a result, unconcealed menstruation becomes inherently unfeminine and socially unacceptable.

Much like objectification, sexism also uses menstruation to marginalize women. Attitudes toward and beliefs about women can be simultaneously hostile and benevolent; this interlocking ideological belief system is referred to as ambivalent sexism (Forbes et al. 2003; Glick and Fiske 1996, 2001). Hostile sexism is the explicit and aggressive or violent prejudice and discrimination against women (for example, rape culture and the belief that women are inferior to men; Forbes et al. 2003; Glick and Fiske 1996). Hostile sexist beliefs and attitudes elicit negative responses toward attempts to dismantle patriarchal systems (Good and Rudman 2010, 482). Benevolent sexism is a way of viewing sex and gender in stereotypical, traditional ways (Forbes et al. 2003; Glick and Fiske 1996). Benevolent sexism implies that menstruation 
is a sign of womanhood and feminine fragility (Forbes et al. 2003; Glick and Fiske 2001), and reinforces the stereotype that women's purpose in life is to bear children. Benevolent sexism can be hard to detect and may not activate negative or hostile feelings, however, it does perpetuate a patriarchal system of gendered power dynamics (Forbes et al. 2003; Glick and Fiske 1996; Good and Rudman 2010). Menstrual leave may then masquerade as a way of protecting the well-being of menstruators, and like benevolent sexism, may still work to undermine menstruators' well-being. An example of benevolent sexism occurring in the context of menstrual leave is evident in Japan's menstrual leave policy, which was created to protect women's capacity to bear children (Dan 1986), and in Zambia's policy, which highlights women's presumed role as mothers/future mothers. In the following sections, we use the framework of objectification and ambivalent sexism to discuss the potential benefits and drawbacks of menstrual leave.

\section{Potential Benefits of Menstrual Leave}

In this section, we consider whether menstrual leave has the potential to positively contribute to the well-being of menstruators in two key interrelated ways: by promoting menstrual health and by de-stigmatizing menstruation.

Menstrual leave may benefit the health of individuals who menstruate, including those who experience mild, moderate, or severe discomfort from menstruation, and those who experience menstrual cycle-related illnesses such as dysmenorrhea, endometriosis, ovarian cysts, and mood disorders. For some women, menstrual symptoms may interrupt their daily lives, making it more difficult to participate in normal activities. For example, in one cross-sectional study with 762 participants, $71.5 \%$ believed that dysmenorrhea was a normal part of women's life, and on average, reported that their menstrual symptoms moderately affected their daily lives (Chen, Kwekkeboom, and Ward 2016, 268-69). However, the social unacceptability of the discussion of menstrual symptoms can result in societal pressure to keep menstrual distress a secret from coworkers and health professionals alike (Johnston-Robledo and Chrisler 2013). Therefore, menstrual leave may offer women the opportunity to speak up about their menstrual cycle-related health issues, and to take time to recover or seek treatment.

Some of the existing menstrual leave policies were clearly written with these positive intentions in mind (for example, Coexist, Gozoop, Culture Machine, and VWT). For example, some policies provide women with the opportunity to take leave or provide workplace flexibility so that women can reserve their medical leave for non-menstrual related illness. Additionally, these policies promote well-being by providing additional opportunities to care for oneself (for example, "well-being rooms," workplace flexibility, normalized discussion of menstruation, et cetera). In our study conducted with a national U.S. sample $(N=600)$, participants were asked what they thought about a menstrual leave policy and the effect the policy would have in the 
U.S.: $23 \%$ of participants believed there would be only positive effects for women in the workplace including time needed to cope with symptoms, and overall improved well-being (Barnack-Tavlaris et al. 2019).

Some proponents of menstrual leave argue that such policies have the potential to benefit women by reducing stigma and encouraging more open discussion of menstruation (CBC Radio 2017). For example, as discussed earlier, at Coexist, female employees are encouraged to discuss menstruation with their line managers (Quarshie 2017) and menstrual leave is discussed in employee meetings among all staff members (Owen 2018; Quarshie 2017). Discussion of menstruation has the potential to be framed in a light that normalizes or neutralizes it, and therefore de-stigmatizes the conversation surrounding it and thus has the potential to reduce (self)objectification and the objectifying gaze of others.

Another positive implication for menstrual leave may aid individuals who identify as gender queer ${ }^{2} /$ non-binary or as transmen. If offered this type of leave, gender queer/non-binary individuals and transmen may feel an increased amount of safety because menstruation may be a time in which these individuals face increased amounts of transphobia and other types of gender discrimination. Chrisler and colleagues (2016) examined transmen's attitudes toward menstruation, and found that some participants reported that they do not feel safe and/or comfortable using a public restroom when they are menstruating because they fear that menstruation will no longer be concealed. If their menstrual status is revealed, this can result in both discrimination and violence. By having the option to use menstrual leave, transmen and individuals who identify as gender queer/non-binary may be able to avoid having their menstrual status revealed (that is, 'outed,' discussed in more depth below).

\section{Potential Drawbacks of Menstrual Leave}

Though menstrual leave may benefit menstruators in some ways, it may also produce a number of unintended negative implications for menstruators, such as perpetuating sexist beliefs and attitudes, contributing to menstrual stigma and perpetuating gender stereotypes, negatively impacting the gendered wage gap, and reinforcing the medicalization of menstruation.

\section{Perpetuating Sexist Beliefs and Attitudes}

Both hostile and benevolent sexism have been found to result in evaluating a menstruating woman more negatively than a non-menstruating woman. For example, Roberts and colleagues $(2002,136)$ found that participants rated a woman more negatively (for example, less competent, less likeable, and more irrational) when they believed that she was menstruating. Furthermore, Forbes and colleagues $(2003,60-61)$ found that both female and male college participants rated a menstruating woman as more impure, less sexually 
desirable, more irritable, and rated her higher on adjectives associated with neuroticism than non-menstruating women. By extension, workers who take advantage of menstrual leave policies could be perceived more negatively than those who do not use the policy. This can be explained by benevolent and hostile sexism which assume that women do not belong in the workplace. Additionally, benevolent sexism may undermine activist intentions by pacifying intentions for progressive change (Becker and Wright 2011, 62, 74). As a result, benevolent sexist attitudes and beliefs may undermine women's and other menstruators' desire to change existing gendered power dynamics.

\section{Contributing to Menstrual Stigma and 'Outing' in the Workplace}

Menstrual leave brings menstruation to light in the workplace. As such, it violates the cultural mandate to keep menstruation concealed. Thus, those who opt to use menstrual leave may put themselves at risk because they may violate cultural norms about menstrual nondisclosure. This unmasking of menstruation in public spaces might contribute to rather than dismantle menstrual stigma. Menstrual leave may also perpetuate the belief that menstruation is something about which to be ashamed and to be kept private, and therefore avoided in the workplace.

The term 'outing' is commonly used to refer to an occurrence in which a member of the LGBTQ+ community's gender identity or sexual orientation is revealed by another person or entity without their permission (Hunter 2007, 154), which may have a number of negative consequences including discrimination, shaming, and violence. It can also be used to refer to the disclosure of one's menstrual status without the menstruator's consent. Enforcing a work place practice concerning menstruation may require "explicit and public definitions for matters that usually remain implicit and private" (Dan 1986, 2). When an employee chooses to use their leave, at the very least, their supervisor will know that they are menstruating, a disclosure that could activate objectification, sexism, and discrimination, even if at an implicit level.

It is also important to acknowledge the diverse and intersectional way in which prejudice and discrimination play out, which may prevent all menstruators from having equal access to menstrual leave. Not all menstruators are women, therefore, fair policies would need to include queer individuals. At the same time, non-binary, gender queer, and trans menstruators may be uncomfortable disclosing their menstrual status; doing so may even result in endangerment (for example, violence toward queer individuals). As such, menstrual leave policies could 'out' queer individuals who must disclose their menstrual status to use the leave provided, or it may, conversely, prevent them from feeling entitled to using menstrual leave. Additionally, there is no current information on whether menstrual leave has been or will be offered to menstruators who do not identify as women. Therefore, fair policies must be inclusive and provide options for confidential disclosure. 


\section{Gender and Menstruation in the Workplace}

Due to societal expectations of women related to childcare and domestic duties, their absence from work is often ascribed to their gender role (Patton and Johns 2007). Thus, menstrual leave may have the potential to perpetuate the assumptions that women are unfit for the workplace and thus, justify gender discrimination. Women may actively choose not to use menstrual leave (if provided) in an attempt to gain workplace advancement (that is, promotions, job training, and social networks) and avoid negative social judgments (Grose and Grabe 2014). For example, it has been reported that women in China are hesitant to use menstrual leave days because they fear that the disclosure of their menstrual status could reinforce stereotypes of female fragility and unproductivity (Forster 2016).

In our study on the anticipated effects of menstrual leave, $49.3 \%$ of participants thought the policy would have only negative effects in the U.S. including discrimination and creating division between genders. Furthermore, $20.7 \%$ of participants believed such policies were unfair to men, or that people would perceive them as unfair to men (Barnack-Tavlaris et al. 2019).

As a result of the same dynamics, menstrual leave may also contribute to the gendered wage gap and the lack of women's advancement in the workplace, a.k.a, 'the glass ceiling.' As previously discussed, researchers have found that women's absence from work perpetuates stereotypes of women as less worthy and reliable employees (Patton and Johns 2007, 1587-601), thus undermining their career development progress.

Men could even use menstruators' absence to their own advantage, undermining menstruators' success in the workplace. In a study on the gendered impact of long working hours on managers, Simpson $(1998,37)$ found that men purposefully use women's absence from work to progress their own position; this creates a competitive masculine culture that purposefully excludes, silences, and appropriates women's work and gives women a tokenized status in the workplace. Simpson $(1998,43)$ also found that men used women's absences as a way to make women appear less committed to their jobs, which helped men increase their own workplace resources while simultaneously hindering the achievements of their female coworkers.

\section{The Medicalization of Menstruation}

The medicalization of menstruation, which portrays menstruation as a disease, contributes to convincing women that their bodies are in need of 'fixing' with the use of various pharmaceutical products (Stein Deluca 2017, 37-38). The medicalization of menstruation also objectifies the female body by commodifying it through capitalist maintenance and care. The patriarchy profits off the menstruating body by making menstruators believe that they need to buy specific menstrual cycle-related products (for example, 
diuretics for bloating and high tech menstrual product innovations such as super absorbent underwear or scented menstrual pads and tampons) for their bodies to remain and/or become socially acceptable, as such furthering the objectification of women.

Through the medicalization of menstruation, menstrual leave may perpetuate the idea that menstruation is 'debilitating' for all or most women, and thus women are not capable of working (efficiently or at all) while menstruating. Menstrual leave may reinforce beliefs that menstruation is an illness that impedes all women, making it appear as a health issue that is associated with both symptoms and risks (Barnack-Tavlaris 2015). In Barnack-Tavlaris et al.'s (2019) study, some participants resisted the idea that menstruation is debilitating, and used that argument to explain why they would not support such a policy. For example, approximately $11 \%$ of participants' qualitative responses pertained to menstrual leave being unnecessary (for example, menstruation is not a disease and should not be treated as such, and that such policies were "ridiculous"). On the other hand, one could argue that creating a separate category of menstrual leave that distinguishes it from medical or sick leave could combat the medicalization of menstruation.

\section{Implementing Menstrual Health Policies and Alternatives}

The potential implications of menstrual leave may be determined by how a policy is both worded and implemented; that is, the intentions that underlie the creation of such a policy can determine the outcome. For example, discussion about menstruation among all employees may foster support for women's reproductive health and reproductive rights, and may help educate people about menstruation since so many misconceptions circulate throughout heteropatriarchal culture (such as the "hormone myth," the idea that women became hormonal monsters when menstruating, and demonstrate characteristics such as unreliable decision-making; Stein Deluca 2017, 1-7). Additionally, discussion of menstruation may produce more positive, or at least neutral, attitudes toward menstruation that do not elicit sexist attitudes, and this may in turn reduce self-objectification (Johnston-Robledo et al. 2007). Therefore, the language used in menstrual leave policies must be created with the intention of ensuring that the policy fosters normalization and open discussion of menstruation, and does not perpetuate objectification, sexism, and patriarchal misconceptions about menstruation. Among the examples presented above, it is apparent that Coexist, Gozoop, Culture Machine, and VWT used progressive feminist values to create their policies in order combat menstrual stigma, sexism, and objectification.

While the effects of menstrual leave policies on menstruator's overall well-being are unclear and in large part depend on how policies are developed and implemented, what seems evident is that some menstruators would benefit from workplace flexibility more generally (for example, more time off, the ability to work from home, customized work schedules). The 
proclaimed need for menstrual leave may be an opportunity for some societies and employers to reevaluate attitudes surrounding absenteeism and work ethic, and consider the creation of sick, parental, and vacation leave policies that are responsive to worker needs, including the provision of additional paid leave. Indeed, in order for any type of leave (that is, menstrual, sick, parental, et cetera.) to effectively aid employee's well-being, some countries may need to reshape their attitudes toward workplace productivity and presenteeism (working while sick).

In one study of Dutch workers, Hansen and Anderson $(2008,956)$ found that over $70 \%$ of employees worked while sick at least once over the course of a year. The researchers note that possible predictors of presenteeism include employment conditions (such as job insecurity) and financial need (Hansen and Anderson 2008, 958), which implies that employees must choose financial stability and job security over their health and well-being. Individuals should not feel pressured to attend work when their health and wellness are at risk. Many cultures stress presenteeism and hyper-productivity in the workplace over health and well-being, which can endanger employees' health (Callen, Linley, and Niederhauser 2013, 1316). In workplaces that stress these qualities, the ideal employee becomes defined as someone who is always available to their employer and does not "bring family concerns or other outside matters to work" (Stein Deluca 2017, 74).

Alternatives to menstrual leave to ensure menstruators' well-being may include equipping workplaces with rest/break rooms for anyone who is feeling under the weather-physically, mentally, or emotionally, such as "well-being rooms," so that employees can "take some time away from work, whether this is for a cup of tea on the sofa or an hour away from their desk to rest . . or to work in a quiet space away from the busy office environment, if needed" (Quarshie 2017). Relatedly, workplaces should consider stocking bathrooms or breakrooms with menstrual products, hot pads, and pain relievers.

\section{Conclusion}

There is a paucity of research that explores the ways in which menstrual leave may potentially benefit or disadvantage menstruators in the workplace, but plenty is known about how gender oppression shapes perceptions of menstruators. As researchers explore the dimensions of menstrual leave policies, we recommend a number of considerations. First, future research must be intersectional and attuned to diverse menstruators and varied menstrual experiences. Second, it should examine the extent to which menstrual leave reinforces the medicalization of menstruation. For example, does the presence of menstrual leave policies inadvertently pressure menstruators to either medically suppress their cycles or avoid taking leave? Further, do coworkers construe a woman's behaviors differently and more negatively (for example, by attributing emotions or behaviors to menstruation/PMS) when she takes menstrual leave? In order to create a policy that fully contributes to 
menstruators' well-being rather than undermining it, menstrual stigma and myths must be challenged and dismantled actively both inside and outside of the workplace.

Assessing the pros and cons of menstrual leave can serve as an entry point to discussions about workplace culture and accommodations more generally. Are sick leave and parental leave provisions ample? How else can workplaces be designed with diverse worker needs in mind? It is important to consider if flexibility can be extended to other stigmatized health conditions (for example, irritable bowel syndrome, mental health disorders, HIV), so that the menstrual policies do not perpetuate discrimination. In advance of a body of research, companies and organizations who choose to implement menstrual leave policies should consider including menstrual discrimination in their anti-discrimination policies, along with training.

In order for menstrual leave policies to be fully beneficial to menstruators' well-being, cultural beliefs about and attitudes toward menstruation must change. Menstrual stigma must be continuously challenged and heteropatriarchal beliefs dismantled. Menstrual leave policies will only advance gender equality if they are adopted in spaces committed to challenging menstrual stigma and dismantling gender-based oppression.

\section{Notes}

1. The term 'menstruator' is used with purpose; this term acknowledges that people who menstruate are not all women, and that not all women menstruate.

2. Queer is an umbrella term used to recognize and include the many identities within the gender spectrum.

\section{REFERENCES}

Barnack-Tavlaris, Jessica L. 2015. "The Medicalization of the Menstrual Cycle: Menstruation as a Disorder." In The Wrong Prescription for Women: How Medicine and Media Create a 'Need' for Treatments, Drugs, and Surgery, edited by Maureen C. McHugh and Joan C. Chrisler, 61-75. Santa Barbara: Praeger.

Barnack-Tavlaris, Jessica L., Kristina Hansen, Rachel B. Levitt, and Michelle Reno. 2019. "Taking Leave to Bleed: Perceptions and Attitudes toward Menstrual Leave Policy." Health Care for Women International 40 (12): 1355-1373. https://doi. org/10.1080/07399332.2019.1639709.

Bartky, Sandra L. 1988. "Foucault, Femininity, and the Modernization of Patriarchal Power." In Femininity and Foucault: Reflection of Resistance, edited by I. Diamond and L Quinby, 93-111. Boston: Northeastern University Press.

Becker, Julia C., and Stephen C. Wright. 2011. "Yet Another Dark Side of Chivalry: Benevolent Sexism Undermines and Hostile Sexism Motivates Collective Action for Social Change." Journal of Personality and Social Psychology 101 (1): 62-77.

Blush Originals. 2017. "First Day of Period Leave [Video File]." Retrieved from https://www.youtube.com/watch?time_continue=11\&v=avPgUxGClSg. 
Bordo, Susan. 1993. "The Body and the Reproduction of Femininity." In Unbearable Weight: Feminism, Western Culture, and the Body, edited by Susan Bordo, 309-26. Berkeley: University of California Press.

Callen, Bonnie L., Lisa C. Lindley, and Victoria P. Niederhauser. 2013. "Health Risk Factors Associated with Presenteeism in the Workplace." Journal of Occupational and Environmental Medicine 55 (11): 1312-17.

$C B C$ Radio. 2017. "Should Women Get Paid Menstrual Leave?" CBC Radio. Retrieved from https://www.cbc.ca/radio/thecurrent/the-current-for-june-1420171.4158414/should-women-get-paid-menstrual-leave-1.4158421.

Chang, Chueh, Fen-Ling Chen, Chu-Hui Chang, and Ching-Hui Hsu. 2011. "A Preliminary Study on Menstrual Health and Menstrual Leave in the Workplace in Taiwan." Taiwan Gong Gong Wei Sheng Za Zhi 30 (5): 436-50.

Chen, Chen X., Kristine L. Kwekkeboom, and Sandra E. Ward. 2016. "Beliefs about Dysmenorrhea and Their Relationship to Self-Management." Research in Nursing of Health 39: 263-76. https://doi.org/10.1002/nur.21726.

Chrisler, Joan C., Jennifer A. Gorman, Jen Manion, Michael Murgo, Angela Barney, Alexis Adams-Clark, Jessica R. Newton, and Meaghan Mcgrath. 2016. "Queer Periods: Attitudes toward and Experiences with Menstruation in the Masculine of Centre and Transgender Community." Culture, Health, \& Sexuality 18 (11): 1238-50.

Dan, Alice. 1986. “The Law and Women's Bodies: The Case of Menstruation Leave in Japan." Healthcare for Women International 7 (1-2): 1-14.

Forbes, Gordon B., Leah E. Adams-Curtis, Kay B. White, and Katie M. Holmgren. 2003. "The Role of Hostile and Benevolent Sexism in Women's and Men's Perceptions of the Menstruating Woman.” Psychology of Women Quarterly 27 (1): $58-63$.

Forster, Katie. 2016. “Chinese Province Grants Women Two Days 'Period Leave' a Month." Independent. Retrieved from http://www.independent.co.uk/news/ world/asia/chinaperiod-leave-ningxia-womentwodays-a-month-menstruation-a7197921.html.

Fredrickson, Barbara L., and Tomi-Ann Roberts. 1997. "Objectification Theory: Toward Understanding Women's Lived Experiences and Mental Health Risks." Psychology of Women Quarterly 21 (2): 173-206.

Good, Jessica J., and Laurie A. Rudman. 2010. "When Female Applicants Meet Sexist Interviewers: The Costs of Being a Target of Benevolent Sexism." Sex Roles 62(78): 481-93.

Glick, Peter, and Susan T. Fiske. 1996. "The Ambivalent Sexism Inventory: Differentiating Hostile and Benevolent Sexism." Journal of Personality and Social Psychology 70 (3): 491-512.

- 2001. "An Ambivalent Alliance: Hostile and Benevolent Sexism as Complementary Justifications for Gender Inequality." American Psychologist 56: 109-18.

Grose, Rose G., and Shelly Grabe. 2014. "Sociocultural Attitudes Surrounding Menstruation and Alternative Menstrual Products: The Explanatory Role of Self-Objectification." Healthcare for Women International 35 (6): 677-94.

Hansen, Claus D., and Johan H. Andersen. 2008. "Going Ill to Work-What Personal Circumstances Attitudes and Work-Related Factors Are Associated with Sickness Presenteeism?” Social Science \& Medicine 67 (6): 956-964. 
Hunter, S. 2007. Coming Out and Disclosures: LGBT Persons across the Life Span. Binghamton, NY: The Haworth Press, Inc.

Johnston-Robledo, Ingrid, and Joan C. Chrisler. 2013. "The Menstrual Mark: Menstruation as Social Stigma." Sex Roles 68 (1-2): 9-18.

Johnston-Robledo, Ingrid, Kristin Sheffield, Jacqueline Voight, and Jennifer Wilcox-Constantine. 2007. "Reproductive Shame: Self-Objectification and Young Women's Attitudes toward Their Reproductive Functioning." Women \& Health 46 (1): 25-39.

Matchar, Emily. 2014. "Should Paid 'Menstrual Leave' Be a Thing?" The Atlantic. Retrieved from https://www.theatlantic.com/health/archive/2014/05/shouldwomen-get-paid-menstrual-leave-days/370789/.

"Labor Standards Act". 1947. Accessed February 24, 2019. Japan. https://www. ilo.org/dyn/travail/docs/2021/Labor\%20Standards\%20Act\%20-\%20www.cas. go.jp\%20version.pdf.

Melican, Casimira, and Grace Mountford. 2017. "Why We've Introduced a Menstrual Policy and You Should Too [Web Log Post].” Victorian Women's Trust. Retrieved from https://www.vwt.org.au/blog-menstrual-policy/.

MH Hub. n.d.a. "Mexico: Agreement by Which Public Servants Are Granted a One-Day Leave of Absence Due to Complications of a Physiological Nature". Accessed February 24, 2019. https://mhhub.org/hive/policy/menstrual-leave/ recMpMmKwVUJsgWWd/.

— n.d.b. "Chile: Menstrual Law." Accessed February 24, 2019. https://mhhub. org/hive/policy/menstrual-leave/recT8plalLMHmAV6K/.

Momigliano, Anna. 2017. "Italy Set to Offer 'Menstrual Leave' for Female Workers." The Independent. Retrieved from https://www.independent.co.uk/news/world/ europe/italy-menstrual-leave-reproductive-health-women-employment-a7649636. html.

Owen, Lara. 2018. "Menstruation and Humanistic Management at Work: The Development and Implementation of a Menstrual Workplace Policy." e-Organizations \& People 25 (4): 23-31.

Patton, Eric, and Gary Johns. 2007. "Women's Absenteeism in the Popular Press: Evidence for a Gender-Specific Absence Culture." Human Relations 60 (11): 1579-612.

Quarshie, Adam. 2017. "Coexist Pioneering Period Policy [Web Log Post]." Hamilton House. Retrieved from https://www.hamiltonhouse.org/coexistpioneering-period-policy/.

Roberts, Tomi-Ann, Rachel M. Calogero, and Sarah J. Gervais. 2018. “Objectification Theory: Continuing Contributions to Feminist Psychology." In APA Handbook of the Psychology of Women: Vol. 1: History, Theory, and Battlegrounds. Washington, DC: American Psychological Association.

Roberts, Tomi-Ann, Jamie L. Goldenberg, Cathleen Power, and Tom Pyszcynski. 2002. "'Feminine Protection': The Effects of Menstruation on Attitudes towards Women." Psychology of Women Quarterly 6: 131-39.

Simpson, Ruth. 1998. "Presenteeism, Power, and Organizational Change: Long Hours as a Career Barrier and the Impact on Working Lives of Women Managers." British Journal of Management 9: 37-50.

Stein Deluca, Robyn. 2017. The Hormone Myth: How Junk Science, Gender Politics \& Lies about PMS Keep Women Down. Oakland: New Harbringer Publications, Inc. 
Worley, Will. 2017. “The Country Where All Women Get a Day Off Because of Their Period." Independent. Retrieved from https://www.independent.co.uk/news/ world/africa/zambia-period-day-off-women-menstruation-law-gender-womens-rights-a7509061.html.

Open Access This chapter is licensed under the terms of the Creative Commons Attribution 4.0 International License (http://creativecommons.org/licenses/ by/4.0/), which permits use, sharing, adaptation, distribution and reproduction in any medium or format, as long as you give appropriate credit to the original author(s) and the source, provide a link to the Creative Commons license and indicate if changes were made.

The images or other third party material in this chapter are included in the chapter's Creative Commons license, unless indicated otherwise in a credit line to the material. If material is not included in the chapter's Creative Commons license and your intended use is not permitted by statutory regulation or exceeds the permitted use, you will need to obtain permission directly from the copyright holder.

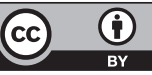

